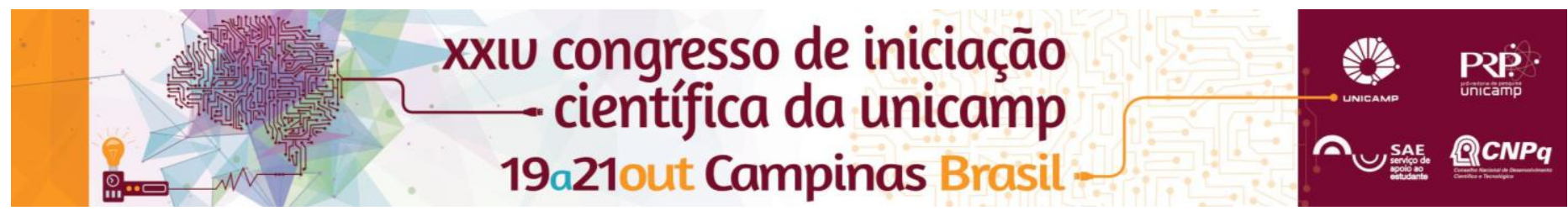

\title{
ENERGY EXPENDITURE DURING AND AFTER TWO RESISTANCE TRAINING PROTOCOLS WITH SAME HYPERTROPHIC GAIN AND INTENSITY BUT DIFFERENT VOLUMES
}

\author{
Enrico A. R. Finardi, Diego T. Brunelli, Ivan L. P. Bonfante, Arthur F. Gáspari, Thiago M. F. Souza, Mara Patricia \\ T. Chacon-Mikahil; Claudia R. Cavaglieri
}

\begin{abstract}
Recently it was observed that two resistance training (RT) protocols performed with $80 \%$ of 1 maximum repetition (1RM) or $30 \%$ of $1 \mathrm{RM}$ until volutional fatigue had the same hypertrophic gains after 10 weeks of training. However, the number of repetitions performed in the protocol with $30 \%$ of $1 \mathrm{RM}$ is higher as compared to the $80 \%$ of $1 \mathrm{RM}$, which leads us to wonder if both protocols can generate the same energy expenditure (EE) during and after the session. In this sense, the purpose of this research was to evaluate the EE during and after two acute RT protocols with same intensity and hypertrophic gains but different volumes. Eleven young male adults $(21.82 \pm 2.62 \mathrm{yrs} ; 1.75 \pm 0.06 \mathrm{~m}$; $71.80 \pm 7.96 \mathrm{~kg} ; 16.91 \pm 6.11 \%$ body fat) underwent a crossover design of two RT protocols performed with $30 \%$ of $1 \mathrm{RM}$ or $80 \%$ of $1 \mathrm{RM}$ until volutional fatigue. Both protocols were composed by 3 sets of leg extension using the corresponding intensities $(30 \%$ or $80 \%$ of $1 \mathrm{RM})$ until fatigue. Participants were measured for oxygen consumption and peak blood lactate concentration during exercise and 60 minutes postexercise after each bout to determine EE and post-exercise oxygen consumption (EPOC). Also, a control session without exercise and with the same procedures for EE and EPOC was carried out. Significant differences were found for total volume and peak lactate levels. However, no differences were observed for EE between the RT protocols. In conclusion, both protocols $(30 \%$ or $80 \%$ of $1 \mathrm{RM}$ ) until fatigue can promote the same EE during and after the sessions.
\end{abstract}

\section{Key words:}

Energy expenditure, Post-exercise oxygen consumption (EPOC), Resistance Training, Young men.

\section{Introduction}

Many of the recommendations for prevention or treatment of overweight and obesity with exercise have focused on aerobic training (AT) for its ability to expend more energy during the sessions when compared to resistance training (RT), however, it is assumed that the main effect of RT in body composition is a change between fat and muscle mass, where the maintenance of a large muscle mass with RT can reduce the risk factors related to increased adipose tissue (STRASSER et al., 2012). Additionally, it is known that the RT performed with equal loads or greater than $80 \%$ of one repetition maximum (1RM) increase hypertrophic gains and consequently the basal metabolism at rest when compared to protocols with lower intensity (ACMS, 2009a).

Recently, Mitchell et al. (2012) observed that young adults submitted to two RT protocols performed with $80 \%$ of 1 maximum repetition (1RM) or $30 \%$ of $1 R M$ until volutional fatigue had the same hypertrophic gains after 10 weeks of training. However, the authors did not measured the energy expenditure (EE) during and after the protocols, which may be interest for the prescription of a RT protocol that promote gains in muscle mass and high $E E$, since the protocol with $30 \%$ of $1 \mathrm{RM}$ have more mechanical contractions. In this sense, the objective of this research was to verify the EE during and after two acute RT protocols with same intensity and hypertrophic gain but different volumes. Since the RT volume can cause a greater impact on EE of the session and the intensity seems to promote greater impact on EPOC (MEIRELLES \& GOMES, 2004), our hypothesis was that the RT protocol performed with $30 \%$ of $1 \mathrm{RM}$ until fatigue can promote higher EE when compared to the $80 \%$ of 1RM protocol.

\section{Results and Discussion}

\begin{tabular}{|c|c|c|c|c|c|}
\hline & \multirow{2}{*}{$\begin{array}{c}\text { Total } \\
\text { Volume } \\
(\mathrm{kg})\end{array}$} & \multirow[t]{2}{*}{$\begin{array}{l}\text { EPOC } \\
\text { (kcal) }\end{array}$} & \multirow[t]{2}{*}{$\begin{array}{c}\text { Total EE } \\
\text { (kcal) }\end{array}$} & \multicolumn{2}{|c|}{$\begin{array}{c}\text { Lactate } \\
\text { (mmol/L) }\end{array}$} \\
\hline & & & & Pre & Post (peak) \\
\hline $30 \%$ & $\begin{array}{c}2301.41 \pm \\
631.19^{\#}\end{array}$ & $77.02 \pm 9.58$ & $112,62 \pm 11,60^{b}$ & $1.44 \pm 0.67$ & $8.00 \pm 1.54 \# b$ \\
\hline $80 \%$ & $\begin{array}{c}1827.05 \pm \\
690.42\end{array}$ & $80.40 \pm 15.57$ & $111,80 \pm 17,61^{\mathrm{b}}$ & $1.27 \pm 0.63$ & $5.83 \pm 1.95^{\# \mathrm{~b}}$ \\
\hline Con & - & $72.60 \pm 17.57$ & $87,28 \pm 19,79$ & $1.40 \pm 0.73$ & $1.27 \pm 0.60$ \\
\hline
\end{tabular}
\# Signifid
$P \leq 0.05$.

\section{Conclusions}

In conclusion, both RT protocols ( $30 \%$ or $80 \%$ of $1 \mathrm{RM}$ ) until fatigue were able to increase EE as compared to the rest and generated the same EE during and after the sessions. In addition, a care should be taken for the prescription of a $30 \%$ of $1 \mathrm{RM}$ protocol for special populations (such as hypertensive individuals), since this protocol showed more physiological intense. More studies investigating the cardiovascular effects behind these two protocols $(30 \%$ or $80 \%$ of $1 \mathrm{RM}$ until fatigue) should be taken into account.

\section{Acknowledgements}

We gratefully acknowledge: the Exercise Physiology Laboratory (FISEX) and the Faculty of Physical Education (FEF) - UNICAMP for all the support in data collection and analysis; the National Counsel of Technological and Scientific Development (CNPq) for the scholarship provided; the enthusiastic participants.

AMERICAN COLLEGE OF SPORTS MEDICINE (ACSM). Progression models in resistance training. Med Sc Sports Exerc, v.41, n.3, p. 687-708, 2009a.

STRASSER, B.; ARVANDI, M.; SIEBERT, U. Resistance training, visceral obesity and inflammatory response: review of the evidence. Obesity Reviews, v.13, n.7, p. 578-591, 2012.

MITCHELL, C.J.; CHURCHWARD-VENNE, T.A.; WEST, D. D. W.; et al. Resistance exercise load does no determine training-mediated hypertrophic gains in young men. J Appl Physiol, v. 113, n.1, 2012.

MEIRELLES, C.M.; GOMES, P.S.C. Acute effects of resistance exercise on energy expenditure: revisiting the impact of the training variables. Rev Bras Med Esporte, vol. 10, 2004. 